\title{
Uso de sustancias en adolescentes y su asociación con factores de riesgo y protección. Un análisis exploratorio de la encuesta escolar a gran escala de Comunidades Que se Cuidan, Colombia
}

\section{Adolescent substance use and its association with risk and protective factors. An exploratory analysis of the large-scale school survey of Comunidades Que se Cuidan, Colombia}

Pablo Montero Zamora*, María Fernanda Reyes Rodríguez $* *$,***, Francisco Cardozo Macías**, Eric C. Brown*, Augusto Pérez Gómez**, Juliana Mejía Trujillo**, Jennifer Toro**, Mayra Paredes Aguilar**.

\footnotetext{
* Departamento de Salud Pública. Escuela Miller de Medicina, Universidad de Miami. ** Corporación Nuevos Rumbos. Bogotá, Colombia. *** Facultad de Psicología. Universidad El Bosque, Bogotá, Colombia.
}

\section{Resumen}

Communities That Care (CTC) es un sistema preventivo que busca disminuir comportamientos problemáticos en adolescentes. En Colombia, este sistema ha sido adaptado bajo el nombre de Comunidades Que se Cuidan (CQC). Este estudio validó las asociaciones entre los factores de riesgo y protección (FRP) para el uso de sustancias psicoactivas (SPA) medidos por CQC y las prevalencias de consumo de estas en adolescentes colombianos. Entre 2012 y 2016 , se aplicó una encuesta a gran escala en jóvenes de 10 a 19 años $(N=$ 50,946) pertenecientes a 23 comunidades de Colombia. Se analizó de forma transversal la asociación entre los FRP con el consumo de alcohol, cigarrillo, marihuana y otras drogas ilegales en los últimos 30 días, año y alguna vez en la vida. Se realizaron regresiones logísticas, ajustando por edad, sexo y sus interacciones con cada FRP. Todas las asociaciones de los 14 FRP evaluados fueron significativas $(p<$ $.001)$. De los efectos observados, $3,0 \%$ se consideraron efectos muy pequeños $(0,70 \leq \mathrm{OR} \leq 1,43), 51,7 \%$ pequeños $(0,70 \geq \mathrm{OR} \geq 1,43)$, $42,6 \%$ medianos $(0,40 \geq \mathrm{OR} \geq 2,48)$ y $7,1 \%$ grandes $(0,23 \geq \mathrm{OR} \geq$ $4,27)$. Se encontraron asociaciones significativas para edad, sexo y sus interacciones con los FRP para la mayoría de FRP. Los hallazgos demuestran la validez de los FRP estudiados para la planificación, el desarrollo y la evaluación futura de sistemas preventivos comunitarios como CQC, los cuales se basan en datos epidemiológicos para la toma de decisiones locales.

Palabras clave: Factores de riesgo; Consumo de SPA; Adolescentes; Prevención.

\begin{abstract}
Communities That Care (CTC) is a prevention system aimed at reducing antisocial behaviors in adolescents. In Colombia, this system has been developed and adapted under the name of Comunidades Que se Cuidan (CQC). Successful implementation of CQC depends on valid associations between measured risk and protective factors (RPFs) for substance use and substance use outcomes. This study assessed these associations using large-scale, school-based surveys of Colombian youth. A cross-sectional analysis was performed. Data from 23 communities in Colombia were collected between 2012 and 2016 from young people $(N=50,946)$ aged 10 to 19 years. Dichotomous alcohol, cigarette, cannabis, and other illegal drug use outcomes were assessed for past 30-day, past-year, and lifetime use. Logistic regression analyses, adjusting for age, gender, and age by RPF, and gender by RPF interactions, were performed for each RPF. All the associations of the 14 RPF evaluated were statistically significant $(p<.001)$. Regarding observed effect sizes, $3.0 \%$ were considered very small $(0.70 \geq \mathrm{OR} \leq$ $1.43), 51.7 \%$ small $(0.70 \geq \mathrm{OR} \geq 1.43), 42.6 \%$ medium $(0.40 \geq \mathrm{OR} \geq$ $2.48)$ and $7.1 \%$ large $(0.23 \geq \mathrm{OR} \geq 4.27)$. Significant main effects for age and gender, and their interactions with RPFs were found for most RPFs. Findings from this study demonstrate the viability of RPFs for adolescent substance use as focal points for intervention planning, development, and evaluation of community-based prevention systems like CQC that rely on epidemiologic data for local decision making. Keywords: Risk factors; Ssubstance use; Adolescents; Prevention.
\end{abstract}




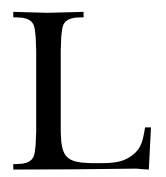

a adolescencia es una etapa en la que existe una alta vulnerabilidad para involucrarse en comportamientos de riesgo, incluyendo el consumo de sustancias psicoactivas (SPA) (Pérez y Scoppetta, 2009). En esta etapa de la vida la persona se encuentra todavía en un periodo de maduración cerebral, por lo tanto, el consumo de SPA es aún más riesgoso (Gruber, Sagar, Dalhgren, Racine y Lukas, 2012; Scoppetta, Pérez y Lanziano, 2011). Se ha encontrado que los adolescentes son más vulnerables a los efectos neurotóxicos del alcohol y las drogas, lo que genera consecuencias negativas a nivel cognoscitivo (Guerri y Pascual, 2010; Zeigler et al., 2005). Así mismo, el abuso de alcohol en edades tempranas está relacionado con un menor volumen en el hipocampo y en la corteza pre-frontal, lo que se asocia con un pobre desempeño a nivel verbal, atencional y viso-espacial (Bellis et al., 2000; Medina, Schweinsburg, Cohen-Zion, Nagel y Tapert, 2007). El fenómeno genera gran preocupación, pues trae consigo problemas escolares, legales, familiares, afectivos y de salud (Espada, Griffin, Botvin y Méndez, 2003; Pérez y Scoppetta, 2009; Wills et al., 2013).

El último estudio de consumo de SPA en población escolar colombiana realizado por el Ministerio de Justicia y del Derecho, Ministerio de Educación Nacional y Ministerio de Salud y Protección Social (2011) mostró que la sustancia con mayor prevalencia de consumo en los jóvenes es el alcohol. Algunas de las conclusiones centrales de este estudio están relacionadas con: (a) una preocupación por hallazgos de edades tempranas de inicio de consumo de sustancias legales e ilegales, (b) una necesidad de contar con un enfoque preventivo para disminuir el uso de alcohol y cigarrillo, y, (c) una necesidad de incrementar las estrategias efectivas de prohibición del expendio de alcohol a los menores de edad.

Ahora bien, es importante tener en cuenta que el consumo de drogas es un fenómeno heterogéneo que cambia a lo largo del tiempo (Glantz, Conway y Colliver, 2005; Sloboda, 2005; Scoppetta et al., 2011; Thatcher y Clark, 2008). Por esto, llevar a cabo una evaluación constante de las prevalencias y su incidencia, así como de los factores de riesgo asociados al consumo es fundamental, pues esto permite orientar las estrategias de prevención e intervención futuras de una manera efectiva (Clayton,1992; Hawkins, Catalano y Miller, 1992; Sloboda, Glantz y Tarter, 2012). En Colombia las iniciativas de prevención desafortunadamente no han estado guiadas por datos epidemiológicos, ni han incluido metodologías sistemáticas para su evaluación. Por ello, identificar los factores de riesgo y protección asociados al consumo de SPA en adolescentes colombianos desde un enfoque científico es una necesidad apremiante.

A principios de los años 90's en los Estados Unidos, Richard F. Catalano y J. David Hawkins desarrollaron el sistema preventivo Communities That Care (CTC) (Hawkins et al., 2008a). CTC tiene como objetivo primordial proveer herramientas para que las comunidades generen y utilicen sus propios datos epidemiológicos acerca de factores de riesgo y protección para el consumo de SPA, los prioricen, e implementen intervenciones eficaces basadas en la evidencia que respondan a factores específicos establecidos como prioritarios (Arthur, Hawkins, Pollard, Catalano y Baglioni, 2002; Hawkins, 2006). Para determinar los perfiles de riesgo y protección para las comunidades CTC desarrolló a su vez el instrumento Communities That Care Youth Survey (CTCYS). Este cuestionario permite realizar de manera sencilla una evaluación diagnóstica del riesgo específico en población adolescente (Arthur et al., 2002; Brown et al., 2009; Hawkins, 2006).

La encuesta original para jóvenes de CTC evalúa 25 factores de riesgo y 13 factores protectores en los dominios: (a) comunidad, (b) escuela, (c) familia, e (d) individuo y pares (Arthur et al., 2002; Hawkins, 2006). Estos factores de riesgo responden a aquellos que se han reportado como predictores de diversos comportamientos problemáticos, como por ejemplo el uso de SPA. (Hawkins et al., 1992; Herrenkohl, Lee, Kosterman y Hawkins, 2012; Kenny y Schreiner, 2009; Kilpatrick et al., 2000). CTC ha demostrado ser eficaz en la reducción de la incidencia y prevalencia del consumo de SPA, violencia y delincuencia juvenil, mediante el abordaje y disminución de los factores de riesgo y protección asociados (FRP) (Feinberg, Greenberg, Osgood, Sartorius y Bontempo, 2007; Feinberg, Jones, Greenberg, Osgood y Bontempo, 2010; Hawkins, et al., 2008a; Hawkins, et al., 2008b; Hawkins, Oesterle, Brown, Abbott y Catalano, 2014; Hawkins et al., 2009; Hawkins et al., 2012; Oesterle et al., 2015). Esto ha llevado a su implementación en otros países como Alemania, Chile, Croacia, Suecia, Australia y los Países Bajos (Toumbourou, 1999; Jonkman et al., 2009).

Por su parte Colombia desde el 2012, inició el proceso de adaptación de CTC bajo el nombre de Comunidades Que se Cuidan (CQC) (Pérez-Gómez, Mejía-Trujillo, Brown y Eisenberg, 2016). La Corporación Nuevos Rumbos ha sido la encargada de llevar a cabo esta labor y durante este tiempo ha logrado adaptar el primer instrumento Latinoamericano derivado de CTC, el cual se enfoca en 18 factores de riesgo y 8 factores protectores, evaluados mediante puntos de corte específicos propios para el país. Sin embargo, como parte del proceso de implementación es preciso llevar a cabo la validación de las asociaciones entre los factores de riesgo y protección (FRP) para el uso de SPA reportados en la literatura y las prevalencias de consumo de estas. Es por esto que el presente trabajo tuvo como objetivo evaluar la asociación y el tamaño de efecto entre los FRP medidos por CQC y el consumo de SPA en adolescentes colombianos, con el fin de garantizar una futura adaptación e implementación exitosa del sistema CQC. 


\section{Método}

\section{Participantes}

La muestra estuvo compuesta por escolares $(N=52,588)$ de $6^{\circ}$ a $11^{\circ}$ grado (Rango de edad $=10$ a 19 años) provenientes de 114 colegios públicos y privados de 23 comunidades de Colombia, pertenecientes a estratos socioeconómicos bajo y medio; todos los estudiantes que estaban presentes el día de la aplicación fueron incluidos en la muestra. De ellos el 52,8\% correspondió al sexo femenino y el 48,2\% al sexo masculino. La media de edad fue 14,2 años (DE= 1,9, rango de 11 a 19). El curso con mayor participación fue el de sexto grado (19,9\%), seguido del de séptimo $(19,1 \%)$, octavo $(18,2 \%)$, noveno $(15,7 \%)$, décimo $(14,7 \%)$ y undécimo $(12,4 \%)$.

\section{Instrumento}

Se utilizó la adaptación del CTCYS de Arthur et al. (2002) en español llamada Encuesta para Jóvenes de Comunidades Que Se Cuidan (EJCQC) (Mejía-Trujillo, Pérez-Gómez y Reyes-Rodríguez, 2015). El instrumento está dirigido a población entre los 10 y 19 años, y está diseñado para ser aplicado en el ámbito escolar. La primera parte del cuestionario recolecta información demográfica y de prevalencia de consumo de alcohol, cigarrillo, marihuana y otras drogas ilegales (cocaína, pasta básica de cocaína, inhalantes, éxtasis, hongos, ácidos, tranquilizantes, popper, anfetaminas, heroína y dick) en el último mes, año y alguna vez en la vida. En una segunda parte, se evalúan 18 de 25 factores de riesgo y 8 de 11 factores de protección evaluados por CTC (Mejía-Trujillo et al., 2015; Pérez-Gómez et al., 2016).

El cuestionario ha mostrado una sensibilidad y especificidad adecuada en población estadounidense y en cinco grupos étnicos incluyendo población latina (Arthur et al, 2007), así como una buena validez predictiva (Briney, Brown, Hawkins y Arthur, 2012). Para la población del estudio, el instrumento cuenta una alta consistencia interna para el cuestionario completo $(\alpha=0,82)$. Respecto a la validez del instrumento la Corporación Nuevos Rumbos realizó una análisis factorial confirmatorio para evaluar la validez de constructo, y los resultados han mostrado índices de ajuste buenos y aceptables para la mayoría de los factores de riesgo y protección (Mejía-Trujillo et al., 2015).

\section{Procedimiento}

Se obtuvo autorización de las Direcciones o Secretarías de Educación de las 23 comunidades situadas en diferentes partes de Colombia. Luego se procedió a obtener el consentimiento informado por parte de los directores de las instituciones educativas, en donde se acordaba y garantizaba la confidencialidad de la información. La encuesta era totalmente anónima y los estudiantes eran advertidos de que su participación era voluntaria y que podían dejar de responder en el momento en que lo desearan.
Posteriormente se realizó la recolección de los datos durante el período de 2012 a 2016. El cuestionario fue suministrado en la jornada escolar por expertos de la Corporación Nuevos Rumbos entrenados previamente. Con el fin de tener más medidas de riesgo dentro de la encuesta, se utilizó la metodología de datos perdidos llamada "diseño de tres cuestionarios" (3-Form Design; Graham, 2012; Little y Rhemtulla, 2013). Este método nos permitió distribuir todos los FRP a través de tres versiones distintas de la encuesta. De esta forma, el número de casos válidos para cada FRP varió según la versión. Si bien todos los FRP no contaron con el mismo número de observaciones, se incluyeron en el análisis solo aquellos que presentaran al menos un $70 \%$ de los casos de la muestra total $(n>36,800)$. Dado que el presente estudio se centró en validar las asociaciones existentes entre los FRP y los comportamientos a través de la interpretación de momios ajustados, y debido a que se contó con una muestra grande, no se consideró necesario realizar imputación múltiple de los datos ausentes.

\section{Análisis de datos}

Los cuestionarios fueron procesados a través de lectura óptica. Se realizó un análisis transversal de la EJCQC utilizando el paquete estadístico STATA 13. Como parte de las estrategias para garantizar la calidad de la información se incluyeron en el instrumento tres preguntas para identificar respuestas de validez cuestionable. De esta manera, aquellos estudiantes que reportaron el uso de una droga ficticia fueron excluidos de la muestra. Fue así como de las 52,588 observaciones iniciales el 3,1\% $(n=1,642)$ fue eliminado de los análisis subsecuentes, por lo que la muestra final estuvo conformada por el 96,9\% $(n=50,946)$ del total inicial.

Se realizó el análisis para 11 de los 18 factores de riesgo y 3 de los 8 factores protectores provenientes de la EJCQC. Esto se debió a que los factores no incluidos fueron incorporados a la EJCQC de forma posterior a este análisis. De esta forma, los 14 FRP (11 factores de riesgo y 3 factores protectores) fueron dicotomizados $(0=$ bajo riesgo o baja protección; 1 = alto riesgo o alta protección) utilizando los puntos de corte diseñados específicamente para CQC y normados para cada grado escolar (Mejía-Trujillo et al., 2015). Así mismo se centraron las medias de cada una de las variables a analizar con el fin de obtener una sola medida de asociación para edad, sexo y sus posibles interacciones y así facilitar la interpretación de los efectos principales. Se utilizó el método de regresión logística para evaluar la asociación entre cada FRP con la prevalencia de consumo de alcohol, cigarrillo, marihuana y otras drogas ilegales en los últimos 30 días, último año y alguna vez en la vida. Bajo estas consideraciones, para evaluar la asociación individual de cada una de las 12 prevalencias de consumo y los 14 FRP se ajustó el siguiente modelo de regresión logística: 
$\log \left[p_{\mathrm{i}} /\left(1-p_{\mathrm{i}}\right)\right]=\beta_{\mathrm{O}}+\beta_{1} F R P c+\beta_{2} E D A D c+\beta_{3} S E X O c+$ $\beta_{4} F R P c_{*} E D A D c+\beta_{5} F R P c_{*} S E X O c$

Donde $p_{\mathrm{i}}$ es igual a la probabilidad de consumo de la sustancia en cuestión de la i-ésima persona, $R P F c$ es la variable centrada del tipo de riesgo o protección según el factor evaluado, $E D A D c$ es la variable centrada de edad en años y $S E X O c$ la variable centrada del sexo. Por su parte $R P F c_{*} E D A D c$ y $R P F c_{*} S E X O c$ corresponden a la variable centrada del tipo de riesgo o protección según la exposición y sus respectivas interacciones con las variables centradas de edad y sexo. Teniendo en cuenta el número de pruebas de hipótesis el análisis fue realizado a partir de valores $p$ ajustados mediante el método de Bonferroni.

La propuesta de análisis respondió a la propuesta conceptual de CTC, que considera que los factores de riesgo tienen la capacidad de influir en el comportamiento de consumo de manera independiente (Arthur et al., 2007). Por ello se consideró que analizar esta asociación en modelos con múltiples factores de riesgo podía traer una situación multicolinealidad entre las covariables. Además, el entender la variabilidad de cada consumo no respondía al objetivo del estudio, el cual era evaluar las asociaciones, su direccionalidad y entender su fuerza de asociación a través de los tamaños de efecto principal de cada FRP.

\section{Consideraciones éticas}

Se contó con un consentimiento informado por parte de los rectores de los colegios, quienes informaban a los padres de familia o representantes legales de los menores bajo la modalidad de consentimiento pasivo, y adicionalmente al momento de la aplicación de la encuesta los estudiantes dieron su asentimiento. Los consentimientos y asentimientos informaban sobre los objetivos del estudio, su carácter confidencial, anónimo y voluntario, el procedimiento de manejo almacenaje anónimo de los datos a través de códigos, así como los posibles riesgos y beneficios. En el salón de clases, se les volvía informar que los datos suministrados en la encuesta eran de carácter confidencial, por lo que no debían registrar ningún tipo de información que llevara a su identificación, y se mencionó que la participación era voluntaria, por lo que cualquiera podría abstenerse de participar, retirarse en cualquier momento y/o solicitar la destrucción del registro si no deseaban ser incluidos en el estudio. El proyecto cuenta con el aval del Comité de Ética de la Corporación Nuevos Rumbos, el cual garantiza el cumplimiento de los principios éticos en investigación que ordena la ley.

\section{Resultados}

\section{Prevalencias de consumo}

En cuanto a las prevalencias de consumo, el alcohol fue la sustancia de mayor consumo $(42,7 \%$ en los últimos 30 días; 70,1\% en el último año; y 73,7\% alguna vez en la vida), seguida del cigarrillo (10,5\% en los últimos 30 días; $21,3 \%$ en el último año; y 26,2\% alguna vez en la vida), la marihuana $(5,1 \%$ en los últimos 30 días; $10,3 \%$ en el último año; y 12,1\% alguna vez en la vida) y la categoría de otras drogas ilegales $(3,7 \%$ en los últimos 30 días; $7,4 \%$ en el último año; y 10,3\% alguna vez en la vida). En general los hombres presentaron prevalencias de consumo mayores que las mujeres especialmente para cigarrillo y marihuana (Tabla 1).

\section{Análisis de los FRP y las prevalencias de consumo}

Se evaluó la asociación de 11 factores de riesgo y 3 factores de protección para cada una de las 12 prevalencias de consumo de SPA. La Figura 1 muestra los efectos principales entre cada FRP individual y las prevalencias de con-sumo de alcohol, cigarrillo, marihuana y otras drogas ilegales (cocaína, basuco, inhalantes, éxtasis, hongos, ácidos, tranquilizantes, popper, anfetaminas, heroína y dick) en las categorías temporales de alguna vez en la vida, año y mes. El efecto fue reportado en razones de momios (OR) ajustadas por edad, sexo y sus interacciones respectivas para cada FRP.

Por otro lado, la Figura 1 muestra una categorización en escala de grises (de blanco a negro). Las celdas en escalas gris oscuro y valores numéricos en blanco representan las posibilidades de desarrollar el comportamiento de riesgo asociado. Las celdas en escalas gris claro y valores numéricos en negro señalan los efectos de amortiguación de los factores protectores estudiados. Las escalas de grises fueron determinadas a través de la conversión a OR de los puntos de corte para la interpretación del tamaño del efecto (Cohen, 1988).

Tabla 1. Prevalencias de consumo de sustancias según sexo y período de tiempo

\begin{tabular}{|c|c|c|c|c|c|c|c|c|c|}
\hline \multirow{2}{*}{ Sustancia } & \multicolumn{3}{|c|}{ Últimos 30 días } & \multicolumn{3}{|c|}{ Últimos 12 meses } & \multicolumn{3}{|c|}{ Alguna vez en la vida } \\
\hline & Femenino & Masculino & Ambos & Femenino & Masculino & Ambos & Femenino & Masculino & Ambos \\
\hline Alcohol & $41,9 \%$ & $41,5 \%$ & $41,7 \%$ & $69,9 \%$ & $70,3 \%$ & $70,1 \%$ & $73,3 \%$ & $74,1 \%$ & $73,7 \%$ \\
\hline Cigarrillo & $8,6 \%$ & $12,3 \%$ & $10,5 \%$ & $18,5 \%$ & $24,0 \%$ & $21,3 \%$ & $23,3 \%$ & $29,0 \%$ & $26,2 \%$ \\
\hline Marihuana & $4,2 \%$ & $6,0 \%$ & $5,1 \%$ & $8,8 \%$ & $11,8 \%$ & $10,3 \%$ & $10,4 \%$ & $13,7 \%$ & $12,1 \%$ \\
\hline Otras drogas ilegales & $2,6 \%$ & $2,8 \%$ & $2,7 \%$ & $7,0 \%$ & $7,8 \%$ & $7,4 \%$ & $9,5 \%$ & $11,2 \%$ & $10,3 \%$ \\
\hline
\end{tabular}




\begin{tabular}{|c|c|c|c|c|c|c|c|c|c|c|c|c|c|}
\hline \multirow[b]{2}{*}{ Dominio } & \multirow[b]{2}{*}{ Factor de riesgo / Factor protector } & \multicolumn{3}{|c|}{$\begin{array}{l}\text { Alcohol } \\
\text { (OR) }\end{array}$} & \multicolumn{3}{|c|}{$\begin{array}{l}\text { Tabaco } \\
(\mathrm{OR})\end{array}$} & \multicolumn{3}{|c|}{$\begin{array}{l}\text { Marihuana } \\
\text { (OR) }\end{array}$} & \multicolumn{3}{|c|}{$\begin{array}{c}\text { Otras dogras ilegales } \\
\text { (OR) }\end{array}$} \\
\hline & & 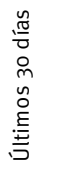 & 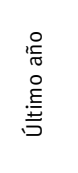 & $\begin{array}{l}\frac{\pi}{\frac{\pi}{2}} \\
\frac{\pi}{\frac{\pi}{5}} \\
\text { 山 }\end{array}$ & 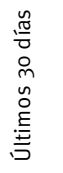 & 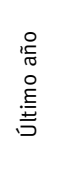 & $\begin{array}{l}\frac{\pi}{2} \\
\frac{\pi}{2} \\
\frac{\pi}{5}\end{array}$ & 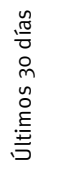 & 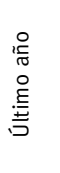 & $\begin{array}{l}\frac{\pi}{2} \\
\frac{\pi}{2} \\
\frac{\pi}{4}\end{array}$ & 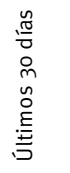 & 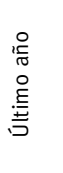 & $\begin{array}{l}\frac{\pi}{3} \\
\frac{\pi}{2} \\
\frac{5}{4}\end{array}$ \\
\hline \multirow{2}{*}{ Comunidad } & Disponibilidad de drogas & 1,57 & 1,70 & 1,75 & 2,92 & 2,59 & 2,56 & 5,06 & 4,17 & 4,02 & 3,03 & 2,65 & 2,65 \\
\hline & Leyes y normas favorables al consumo & 1,56 & 1,55 & 1,56 & 1,87 & 1,74 & 1,75 & 2,01 & 1,84 & 1,82 & 2,10 & 1,70 & 1,76 \\
\hline \multirow{2}{*}{ Escuela } & Bajo compromiso escolar & 1,59 & 1,71 & 1,81 & 2,70 & 2,51 & 2,39 & 2,89 & 2,59 & 2,56 & 2,60 & 2,36 & 2,30 \\
\hline & Reconocimiento en escuela por participación & 0,75 & 0,67 & 0,62 & 0,57 & 0,62 & 0,60 & 0,55 & 0,60 & 0,58 & 0,55 & 0,57 & 0,54 \\
\hline \multirow{5}{*}{ Familia } & Problemas de manejo familiar & 1,66 & 1,64 & 1,59 & 3,09 & 2,71 & 2,57 & 3,51 & 3,08 & 2,93 & 2,80 & 2,52 & 2,50 \\
\hline & Actitud favorable de padres al uso de drogas & 2,93 & 3,62 & 3,86 & 2,48 & 2,27 & 2,22 & 2,81 & 2,41 & 2,32 & 2,35 & 2,19 & 2,12 \\
\hline & Actitud favorable de padres al comportamiento antisocial & 1,66 & 1,86 & 2,01 & 2,71 & 2,54 & 2,53 & 3,11 & 2,90 & 2,80 & 2,62 & 2,73 & 2,61 \\
\hline & Oportunidades familiares para involucrarse & 0,74 & 0,75 & 0,75 & 0,51 & 0,54 & 0,56 & 0,47 & 0,53 & 0,55 & 0,58 & 0,59 & 0,59 \\
\hline & Reconocimiento familiar por participación & 0,74 & 0,69 & 0,68 & 0,50 & 0,54 & 0,55 & 0,46 & 0,49 & 0,52 & 0,54 & 0,56 & 0,56 \\
\hline \multirow{5}{*}{ Individuo y Pares } & Baja percepción de riesgo al consumo de drogas & 2,93 & 1,70 & 1,76 & 2,37 & 2,07 & 2,02 & 4,25 & 3,40 & 3,17 & 3,12 & 2,57 & 2,49 \\
\hline & Actitudes favorables al uso de drogas & 2,41 & 2,82 & 2,91 & 4,45 & 3,64 & 3,53 & 5,59 & 4,47 & 4,27 & 4,16 & 3,57 & 3,31 \\
\hline & Actitudes favorables hacia el comportamiento antisocial & 1,60 & 1,82 & 1,93 & 2,62 & 2,40 & 2,43 & 3,08 & 2,65 & 2,68 & 2,99 & 2,69 & 2,61 \\
\hline & Comportamientos antisociales en amigos & 1,81 & 2,02 & 2,07 & 2,97 & 2,74 & 2,61 & 3,39 & 3,24 & 3,16 & 3,10 & 3,06 & 2,92 \\
\hline & Uso de drogas en amigos & 2,89 & 3,78 & 4,12 & 6,33 & 5,51 & 5,03 & 8,64 & 7,41 & 7,05 & 5,25 & 4,55 & 4,25 \\
\hline
\end{tabular}

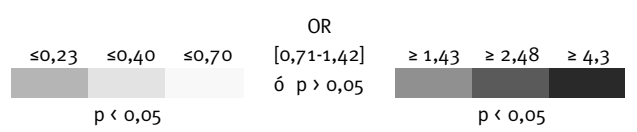

Figura 1. Mapa de calor de las razones de momios ajustadas (OR) entre los factores de riesgo y protectores de la encuesta para jóvenes de CQC según dominio y consumo de alcohol, tabaco, marihuana y otras drogas ilegales, para ambos sexos.

Nota. † OR: Razón de momios ajustada por Edad; Sexo; Edad x [Factor de Riesgo o Factor de Protección]; Sexo x [Factor de Riesgo o Factor de Protección].

Así mismo, aquellos valores de OR de 0,71 a 1,42 fueron considerados como efectos muy pequeños según lo establecido por Sawilowsky (2009). Esta perspectiva permitió observar y comparar cada uno de los 168 efectos independientes obtenidos para cada sustancia y categoría temporal en términos de la fuerza de asociación específica del efecto. Cabe señalar que en caso de que la asociación fuera no significativa estadísticamente o el efecto fuera considerado muy pequeño el valor del OR aparecería en color blanco.

A pesar de que todas las 168 asociaciones evaluadas fueron altamente significativas $(\mathrm{p}<.05)$ las tonalidades de grises variaron solo para el $97 \%$, ya que cinco $(3,0 \%)$ efectos pertenecientes a factores protectores fueron considerados muy pequeños según el criterio establecido $(\mathrm{OR}=[0,71$ $0,99])$. Es importante resaltar que los cinco efectos mencionados anteriormente pertenecen a asociaciones con el consumo de alcohol (Figura 1). De esta forma de los efectos observados, $3,0 \%$ se consideraron efectos muy pequeños $(0,70 \leq \mathrm{OR} \leq 1,43), 51,7 \%$ pequeños $(0,70 \geq \mathrm{OR} \geq$ $1,43), 42,6 \%$ medianos $(0,40 \geq \mathrm{OR} \geq 2,48)$ y $7,1 \%$ grandes
$(0,23 \geq \mathrm{OR} \geq 4,27)$. El dominio que presentó las razones de momios más elevadas para el uso de cualquier sustancia fue el de pares-individuo, seguido del de familia, escuela y comunidad. De manera general, se encontró que la disponibilidad de drogas, la baja percepción de riesgo del uso de drogas, actitudes favorables hacia el uso de drogas y uso de drogas en amigos fueron los predictores más importantes en el consumo de SPA.

\section{Asociaciones en el dominio comunitario}

En el dominio comunitario se analizaron los efectos en el consumo de SPA para dos factores de riesgo: 1) disponibilidad de drogas; y 2) leyes y normas favorables al consumo. En general, la disponibilidad de drogas fue el factor de riesgo más relevante por encima de las leyes y normas favorables al consumo. Se observó un tamaño de efecto mediano en el 66,7\% de las asociaciones entre disponibilidad de drogas y el uso de SPA en los jóvenes en especial para cigarrillo, marihuana y otras drogas ilegales. De los efectos entre leyes y normas favorables al consumo y el uso de SPA 
el $100 \%$ fue considerado pequeño. En particular, la asociación entre la disponibilidad de drogas y el consumo de marihuana fue la que presentó los tamaños de efecto más grandes, aumentando hasta 5 veces la posibilidad de consumo de la droga en los últimos 30 días (OR=5,06; IC95\%: $4,46,5,74)$ en aquellos jóvenes que percibieron como alta su disponibilidad.

Por su parte, la posibilidad de consumo de marihuana en el último año en aquellos jóvenes que reportaron una alta disponibilidad fue 4,17 veces la posibilidad de los que no $(\mathrm{OR}=4,17$; IC95\%: $3,84,4,54)$ y para el consumo alguna vez en la vida 4,02 veces en comparación con los que percibieron baja su disponibilidad ( $\mathrm{OR}=4,02$; IC95\%: 3,72, 4,35). En cuanto a las otras SPA, el conjunto de otras drogas ilegales presentó mayores tamaños de efectos para su utilización en comparación con los tamaños de efecto observados para cigarrillo y alcohol. No se incluyó ningún factor protector en este dominio (Figura 1. Dominio Comunidad).

\section{Asociaciones en el dominio escolar}

En el dominio escolar se analizaron los efectos en el consumo de SPA para un factor de riesgo: 1) bajo compromiso escolar, y un factor protector: 2) actitudes favorables de los padres hacia el consumo de drogas. Se observó un tamaño de efecto mediano en el 50,0\% de las asociaciones entre bajo compromiso escolar y el uso de SPA en los jóvenes. El otro 50,0\% fue considerado bajo. Las asociaciones más relevantes fueron observadas en los jóvenes que reportaron bajo compromiso escolar con consumo en los últimos 30 días de marihuana (OR=2,89; IC95\%: 2,59, 3,22), cigarrillo $(\mathrm{OR}=2,70$; IC95\%: 2,51, 2,90) y otras drogas ilegales (OR=2,60; IC95\%: 2,29, 2,95). Por su parte, el reconocimiento en la escuela por la participación como factor protector mostró en general efectos pequeños. El efecto protector más relevante fue observado para la disminución en un $46 \%$ del consumo de drogas ilegales alguna vez en la vida $(\mathrm{OR}=0,54 ; \mathrm{IC95 \%}: 0,50,0,59), 45 \%$ de reducción para el consumo de otras drogas ilegales en el último mes (OR=0,55; IC95\%: 0,48, 0,64) y marihuana en el último mes (OR=0,55; IC95\%: 0,49, 0,62). El consumo de alcohol fue el que presentó menores efectos protectores por el reconocimiento escolar, en particular su uso en los últimos 30 días (OR=0,75; IC95\%: 0,71, 0,78) fue el menos favorecido por la presencia de este factor protector (Figura 1. Dominio Escolar).

\section{Asociaciones en el dominio familiar}

Se analizaron tres factores de riesgo: 1) problemas en el manejo familiar; 2) actitudes favorables de los padres hacia el uso de drogas; 3) actitudes favorables de los padres hacia el comportamiento problemático; y dos factores de protección: 1) oportunidades familiares para involucrarse; 2) reconocimiento en la familia por la participación. Se observó un tamaño de efecto mediano en el $63,9 \%$ de las asociacio- nes para los tres factores de riesgo y el uso de SPA en los jóvenes. El otro $36,1 \%$ fue considerado pequeño. Las asociaciones más relevantes fueron observadas en los jóvenes que reportaron actitudes favorables de los padres hacia el uso de drogas y consumo de alcohol en el último año $(\mathrm{OR}=3,86$; IC95\%: $3,62,4,11)$ y alguna vez en la vida $(\mathrm{OR}=3,62 ; \mathrm{IC} 95 \%$ : $3,42,3,82)$. Otra asociación a destacar fue entre problemas de manejo familiar y el consumo de marihuana en los últimos 30 días (OR=3,51; IC95\%: 3,14, 3,93).

En cuanto a los factores de protección, más del 83,3\% fue considerado pequeño y el $16,7 \%$ restante muy pequeño. La protección de los individuos que reportaron oportunidades familiares para involucrarse en actividades de tipo prosocial fue considerada en su totalidad como muy baja en el caso específico del consumo de alcohol. La misma situación se observó para la asociación entre el reconocimiento de la familia por la participación en actividades prosociales y el consumo de alcohol en los últimos 30 días. Los efectos protectores más relevantes observados fueron la aparente disminución de un 54,0\% de las posibilidades en el consumo de marihuana en los últimos 30 días (OR=0,46; IC95\%: 0,42, 0,52) en los jóvenes con mayores oportunidades familiares y de un $55,0 \%$ de disminución (OR=0,45; IC95\%: 0,41, 0,52) en el mismo tipo de consumo en los jóvenes que fueron reconocidos por su familia dado su involucramiento en actividades prosociales (Figura 1. Dominio Familia)

\section{Asociaciones en el dominio de pares e individuo}

Se analizaron los factores de riesgo: 1) baja percepción de riesgo del consumo de drogas ; 2) actitudes favorables hacia el uso de drogas; 3 ) actitudes favorables hacia el comportamiento problemático; 4) comportamientos problemáticoes en amigos; 5) uso de drogas en amigos. Como se mencionó anteriormente en este fue el domino en donde se observaron los efectos de mayor tamaño y en mayor número. Un $18,3 \%$ de los efectos analizados fue considerado como grande ( $\mathrm{OR} \geq 4,27), 60,0 \%$ mediano $(\mathrm{OR} \geq 2,48$ ) y solo un $21,7 \%$ como pequeño ( $\mathrm{OR} \geq 1,43$ ). Las asociaciones más grandes para todas las sustancias y categorías temporales, con excepción de alcohol en el último mes, fueron observadas en los jóvenes que reportaron uso de drogas en amigos y tener una actitud favorable hacia su consumo. Particularmente, los OR más elevados de todos se observaron entre el uso de drogas en amigos y el consumo de marihuana en el último mes ( $\mathrm{OR}=8,64$; IC95\%: $7,51,9,24)$, año (OR=7,41; IC95\%: 6,76, 8,11), y alguna vez en la vida $(\mathrm{OR}=7,05$; IC95\%: 6,47, 7,68). Este mismo factor de riesgo fue el más importante en el aumento de las posibilidades de consumo de cigarrillo en el último mes (OR=6,33; IC95\%: 5,81, 6,90), año (OR=5,51; IC95\%: 5,20, $5,82)$ y alguna vez en la vida ( $\mathrm{OR}=, 03$; $\mathrm{IC} 95 \%: 4,77,5,30)$.

Otras asociaciones a destacar relacionadas con el consumo de drogas en amigos fue la del incremento del con- 
sumo de otras drogas ilegales en el último mes $(\mathrm{OR}=5,25$; IC95\%: 4,53, 6,10), año (OR=4,55; IC95\%: 4,17, 4,97) у alguna vez en la vida (OR=4,25; IC95\%: 3,95, 4,59). En cuanto a los otros factores de riesgo las actitudes favorables de los jóvenes hacia el consumo de drogas estuvieron altamente asociadas con el consumo de cigarrillo y marihuana en el último mes y el consumo de marihuana en el último año. Cabe resaltar que el comportamiento problemático en amigos y una actitud favorable por parte de los jóvenes hacia este tipo de comportamientos se asoció en general de manera baja con el consumo de alcohol y media con las demás sustancias (Figura 1. Dominio Pares-Individuo)

\section{Significancia de los predictores}

La Tabla 2 muestra las covariables que se observaron como significativas $(p<.05)$ para la asociación entre cada FRP y el consumo alcohol, cigarrillo, marihuana y otras drogas ilegales alguna vez en la vida. Esta evaluación permitió confirmar la asociación esperada de cada covariable con respecto al consumo de cada sustancia. De las covariables evaluadas la edad se asoció con todas las sustancias. De esta forma la posibilidad de consumo de cualquier sustancia alguna vez en la vida fue más elevada en los estudiantes de mayor edad. Así mismo, la edad tuvo un efecto de interacción sinérgico con la mayoría de FRP para todas las SPA, aumentando su efecto principal. Por su parte el sexo se asoció con el cigarrillo y todas las sustancias ilegales (marihuana y otras sustancias ilegales) y fueron los hombres quienes presentaron mayores posibilidades de consumo alguna vez en la vida. Sin embargo, a pesar de su relevancia como predictor del consumo de cigarrillo y drogas ilegales, el sexo no se asoció al consumo de alcohol. Las interacciones del sexo con cada factor de riesgo fueron más evidentes para el consumo de cigarrillo. Cabe señalar que a nivel de los factores protectores todas las interacciones entre sexo y el factor protector fueron significativas y de tipo sinérgico para todas las sustancias excepto alcohol.

\section{Discusión}

Similar al último estudio nacional de escolares para Colombia, en esta investigación las sustancias de mayor consumo fueron alcohol, cigarrillo y marihuana. Sin embargo, las prevalencias de vida, año y mes para alcohol y cigarrillo fueron superiores a las nacionales. Para el caso específico de la marihuana se encontró que el consumo de esta sustancia fue dos veces mayor al promedio nacional para las tres prevalencias (Ministerio de Justicia y del Derecho, Ministerio de Educación Nacional y Ministerio de Salud y Protección Social, 2011).

Quizá el hallazgo más importante derivado de este estudio fue que se encontró evidencia a favor del conjunto de 14 FRP seleccionados en sus cuatro dominios (familia, comunitario, escolar e individual y pares) como predictores del consumo de SPA en adolescentes colombianos, lo que valida de forma concurrente los FRP de CQC con los presentes y validados en la literatura en términos de direccionalidad y fuerza (Arthur et al., 2007, Briney et al., 2012; Glaser, Van Horn, Arthur, Hawkins y Catalano, 2005; Hawkins et al., 1992). Este hallazgo brinda información re-

Tabla 2. Covariables e interacciones estadísticamente significativas $(p<0,001)$ según FRP y SPA alguna vez en la vida

\begin{tabular}{|c|c|c|c|c|}
\hline Factor de riesgo / Factor protector & Alcohol & Cigarrillo & Marihuana & $\begin{array}{l}\text { Otras drogas } \\
\text { ilegales }\end{array}$ \\
\hline Disponibilidad de drogas & $\mathrm{E}$ & $E, S$, ExR & $E, S, E x R, S x R$ & $\mathrm{E}, \mathrm{S}$ \\
\hline Bajo compromiso escolar & $E, S, S x R$ & E, S, ExR & $E, S$ & $\mathrm{E}, \mathrm{S}$ \\
\hline Reconocimiento en escuela por participación & $\mathrm{E}$ & $E, S$ & $E, S$ & $E, S$ \\
\hline Actitud favorable de padres al uso de drogas & $\mathrm{E}$ & $E, S$ & $E, S$ & $E, S$ \\
\hline Actitud favorable de padres al comp. antisocial & $E$ & $E, S, S x R$ & $E, S, S x R$ & $\mathrm{E}, \mathrm{S}$ \\
\hline Oportunidades familiares para involucrarse & $\mathrm{E}$ & E, S, ExP,SxP & E, S, ExP & E, S, ExP \\
\hline Reconocimiento familiar por participación & $\mathrm{E}$ & E, S, SxP & E, S, ExP,SxP & E, S, ExP \\
\hline Baja percepción de riesgo al consumo de drogas & $\mathrm{E}$ & $\mathrm{E}, \mathrm{S}, \mathrm{S} \times \mathrm{R}$ & $E, S$ & $E$ \\
\hline Actitudes favorables hacia el comp. antisocial & $\mathrm{E}, \mathrm{S} \times \mathrm{R}$ & E, S, SxR & $E, S, S \times R$ & $E, S, S \times R$ \\
\hline Comportamientos antisociales en amigos & $\mathrm{E}$ & $E, S, S \times R$ & $E, S$ & $E, S, S \times R$ \\
\hline Uso de drogas en amigos & $E$ & $E, S$ & $E, S$ & E, ExR \\
\hline
\end{tabular}

Nota. $†$ FRP = factores de riesgo y protección; SPA=sustancia psicoactiva; $E=E$ dad ; $\mathrm{S}=\mathrm{Sexo}$; ExR= Interacción edad y factor de riesgo; ExP= Interacción edad y factor de protección; SxR= Interacción sexo y factor de riesgo; SxP= Interacción sexo y factor de protección; Comp.=comportamiento. 
levante para Latinoamérica, ya que permite asumir que los FRP para el consumo de SPA podrían ser universales. Lo anterior implica que su utilización es relevante y adecuada para la implementación de iniciativas de tipo preventivo que pretendan reducir y monitorear a través del tiempo las prevalencias de consumo adolescente de alcohol, cigarrillo, marihuana y otras drogas ilegales.

Específicamente en el dominio comunitario, la disponibilidad de drogas como factor de riesgo predijo de manera apropiada el alto consumo de sustancias ilegales tanto para el último año como para el último mes; este hallazgo es similar a lo encontrado en el Estudio Nacional de Consumo de Sustancias Psicoactivas en Población Escolar de Colombia, en donde se reportó que una mayor percepción de facilidad para conseguir SPA estuvo relacionada con consumo de estas sustancias (Ministerio de Justicia y del Derecho, Ministerio de Educación Nacional, y Ministerio de Salud y Protección Social, 2011). Esta situación es de especial interés para el contexto colombiano, ya que los menores tienen una percepción generalizada de facilidad de acceso a SPA, lo cual podría estar relacionado con bajos controles sobre la venta a menores en el país, así como con la larga tradición de aceptación de consumo de sustancias como alcohol desde edades tempranas, la cual llevaría a un posible incremento en el consumo de otras sustancias como cigarrillo o drogas ilegales.

Por su parte, la relación entre los factores en el dominio familiar y el consumo de SPA hallada en este estudio, fue encontrada igualmente en el Estudio Nacional de Consumo de Sustancias Psicoactivas en Población Escolar de Colombia (Ministerio de Justicia y del Derecho, Ministerio de Educación Nacional, y Ministerio de Salud y Protección Social, 2011). Esto confirma que el trabajo con las familias es fundamental para la prevención. Hawkins, Catalano y Miller (1992) respaldan la premisa de que los padres deben tener un adecuado manejo familiar basado en las habilidades para establecer límites y normas, disciplina a través de negociación y tener buenas relaciones con los miembros de la familia. Así mismo, la relación entre los padres ejerce influencia en el consumo de SPA por parte de los menores, por lo que las buenas relaciones y la comunicación familiar reflejadas en las oportunidades y reconocimiento familiar actúan como factores protectores reduciendo el riesgo de consumo (Plenum-Sanz, Iraurgi, Martínez y Cosgaya, 2006; Mallick 2009).

En el dominio del individuo y de pares, los resultados mostraron que los factores de riesgo actitudes favorables hacia el uso de drogas, actitudes favorables hacia el comportamiento problemático y uso de drogas en amigos se asociaron fuertemente al consumo frecuente de SPA. Esto corresponde a lo encontrado en otras investigaciones en las que se reportó que estos factores son quizá los mejores predictores para el consumo y pueden ser ejemplificados a partir de la selección de amigos para el consumo (Dishion y Owen, 2002), ofrecimiento de SPA por parte de los pares y la persuasión por parte de amigos y ambientes de consumo (Moral y Ovejero, 2008). Por su parte, el inicio del consumo de alcohol, tal como lo muestran Pérez y Scoppetta (2008) tiende a generarse con el avance de la edad, lo que podría darse con el propósito de explorar, buscar reconocimiento y aceptación de un grupo de pares (Cicua, Méndez y Muñoz, 2008). Con base en esta evidencia, la adopción de abordajes como el propuesto por CTC y ahora por CQC en Colombia es primordial para que las comunidades prioricen los factores de riesgo e intervengan sobre ellos de forma individual para prevenir los comportamientos problemáticos en los jóvenes.

Por otra parte, la gran variabilidad en los tamaños de efecto observados a nivel general puede ser explicada a partir del dinamismo y heterogeneidad del fenómeno de consumo de SPA, el cual como se mencionó antes ha sido reportado como cambiante a lo largo del tiempo (Glantz et al., 2005; Sloboda, 2005; Thatcher y Clark, 2008; Scoppetta et al., 2011) y en el caso específico de este estudio, a lo largo de cada dominio estudiado. Dada esta situación, instrumentos como la EJCQC, el cual no solo mide las prevalencias de consumo de SPA sino también la exposición a los principales factores de riesgo que explican su variabilidad, lo convierten en una herramienta a aplicar a nivel nacional y de manera continua al menos cada dos años según las recomendaciones de CTC (Hawkins, Catalano y Arthur, 2002).

\section{Limitaciones y recomendaciones}

Finalmente, dentro de las limitaciones de este estudio, la principal a nuestro criterio fue el tipo de diseño seleccionado para evaluar asociaciones, ya que al ser transversal se pierde poder explicativo en las relaciones evaluadas y las inferencias se encuentran sujetas a posibles sesgos de causalidad reversa. Sin embargo, dado que estos mismos factores fueron evaluados de manera longitudinal en otros contextos para efectos de este trabajo hemos asumido que su comprobada universalidad también permite confirmar la temporalidad de la relación y por ende desestimar el sesgo. A pesar de esto, se recomienda continuar reportando futuros hallazgos a través del uso de mediciones longitudinales que permitan tener una evaluación sistemática, dinámica y continua de las comunidades, resaltando los cambios o estabilidad de los factores de riesgo que se presentan, para direccionar estrategias de prevención aún más efectivas y mejor dirigidas. Así mismo, es importante mencionar que las mediciones fueron obtenidas mediante el autoinforme por parte de las personas encuestadas. Sin embargo, gracias a la rigurosidad con la que se aplicaron los instrumentos se considera que en este caso el autoinforme fue un método adecuado y directo para la evaluación de las respuestas cognitivas y la experiencia subjetiva del individuo. 


\section{Conclusiones}

Esta investigación es de las pocas en su tipo en informar acerca de los perfiles de riesgo y el comportamiento asociado al consumo de SPA en Colombia utilizando un enfoque epidemiológico a partir de indicadores de riesgo reportados en la evidencia y ahora validados para el país.

Los hallazgos de este estudio mostraron la validez de los FRP estudiados como puntos de partida para la planificación, el desarrollo y la evaluación futura de intervenciones llamadas a disminuir el consumo de SPA en Colombia. Así mismo, los resultados resaltan la importancia del uso de sistemas preventivos comunitarios como CQC, los cuales se basan en datos epidemiológicos para la toma de decisiones locales.

\section{Reconocimientos}

Agradecemos el financiamiento de este estudio al Instituto Nacional sobre el Abuso de Drogas (NIDA, \# DA031175). También agradecemos a todas las comunidades y escuelas participantes de las 23 comunidades de Colombia.

\section{Conflictos de interés}

No se tienen conflictos de interés.

\section{Referencias}

Arthur, M. W., Briney, J. S., Hawkins, J. D., Abbot, R. D., Brooke-Weiss, B. L. y Catalano, R. F. (2007). Measuring risk and protection in communities using the Communities That Care Youth Survey. Evaluation and Program Planning, 30, 197-211. doi:10.1016/je.evalprogplan.2007.01.009.

Arthur, M. W., Hawkins, J. D., Pollard, J. A., Catalano, R. F. y Baglioni A. J., Jr. (2002). Measuring risk and protective factors for substance use, delinquency and other adolescent problems behaviors: The Communities That Care Youth Survey. Evaluation Review, 24, 575-601. doi:10.1177/019384102237850.

De Bellis, M. D., Clark, D. B., Beers, S. R., Soloff, P. H., Boring, A. M., Hall, J., ... Keshavan, M. S. (2000). Hippocampal volume in adolescent-onset alcohol use disorders. American Journal of Psychiatry, 157, 737-744. doi:10.1176/appi.ajp.157.5.737.

Briney, J. S., Brown, E. C., Hawkins, J. D. y Arthur, M. W. (2012). Predictive validity of established cut points for risk and protective factor scales form the Communities That Care Youth Survey. Journal of Primary Prevention, 33, 249-258. doi:10.1007/s10935-012-0280-1.

Brown, E. C., Graham, J. W., Hawkins, J. D., Arthur, M. W., Baldwin, M. M., Oesterle, S. y Abbott, R. D. (2009). Design and analysis of the Community Youth Development
Study longitudinal cohort sample. Evaluation Review, 33, 311-334.

Clayton, R. R. (1992). Transitions in drug use: Risk and protective factors. In M. D. Glantz y R. W. Pickens (Eds.), Vulnerability to drug abuse (pp. 15-51). Washington, DC: American Psychological Association. doi:10.1037/10107-001.

Cohen, J. (1988). Statistical power analysis for the behavioral sciences (2nd Ed.). Hillsdale, NJ: Erlbaum Associates.

Crow, I., France, A., Hacking, S. y Hart, M. (2004). Does Communities That Care work? An evaluation of a community-based risk prevention programme in three neighbourhoods. York: Joseph Rowntree Foundation.

Dishion, T. J. y Owen, L. D. (2002). A longitudinal analysis of friendships and substance use: Bidirectional influence from adolescence to adulthood. Developmental Psychology, 38, 480-491. doi:10.1037//0012-1649.38.4.480.

Espada, J. P., Botvin, G. J., Griffin, K. W. y Méndez, X. (2003). Adolescencia: Consumo de alcohol y otras drogas. Papeles del Psicólogo, 23, 9-17.

Feinberg, M. E., Greenberg, M. T., Osgood, D. W., Sartorius, J. y Bontempo, D. (2007). Effects of the Communities That Care model in Pennsylvania on youth risk and problem behaviors. Prevention Science, 8, 261-270.

Feinberg, M. E., Jones, D., Greenberg, M. T., Osgood, D. W. y Bontempo, D. (2010). Effects of the Communities That Care model in Pennsylvania on change in adolescent risk and problem behaviors. Prevention Science, 11, 163-171.

Glantz, M. D., Conway, K. P. y Colliver, J. D. (2005). Drug abuse heterogeneity and the search for subtypes. In Z . Sloboda (Ed.). Epidemiology of drug abuse (pp. 15-28). New York, NY: Springer.

Glaser, R. R., Van Horn, M. L., Arthur, M. W., Hawkins, J. D. y Catalano, R. F. (2005). Measurement properties of the Communities That Care Youth Survey across demographic groups. Journal of Quantitative Criminology, 21, 73-102.

Graham, J. W. (2012). Missing data: Analysis and design. New York, NY: Springer. doi:10.1007/978-1-46144018-5_1

Gruber, S. A., Sagar, K. A. Dahlgren, M. K., Racine, M. y Lukas, S. E. (2012). Age of onset of marijuana use and executive function. Psychology of Addictive Behaviors, 26, 496-506. doi:10.1037/a0026269.

Guerri, C. y Pascual M. (2010). Mechanisms involved in the neurotoxic, cognitive, and neurobehavioral effects of alcohol consumption during adolescence. Alcohol, 44, 15-26. doi:10.1016/j.alcohol.2009.10.003.

Hawkins, J. D. (2006). Science, social work, prevention: Finding the intersections. Social Work Research, 30, 137-152.

Hawkins, J. D., Catalano, R. F. y Miller, J. Y. (1992). Risk and protective factors for alcohol and other drug problems in adolescence and early adulthood: Implications for substance abuse prevention. Psychological Bulletin, 112, 64-105. 
Hawkins, J. D. Catalano, R. F. y Arthur, M. W. (2002). Promoting science-based prevention in communities. Addictive Behaviors, 27, 951-976.

Hawkins, J. D., Catalano, R. F., Arthur, M. W., Egan, E., Brown, E. C., Abbott, R. D. y Murray, D. M. (2008a). Testing Communities That Care: The rationale, design and behavioral baseline equivalence of the Community Youth Development Study. Prevention Science, 9, 178-190.

Hawkins, J. D., Brown, E. C., Oesterle, S., Arthur, M. W., Abbott, R. D. y Catalano, R. F. (2008b). Early effects of Communities That Care on targeted risks and initiation of delinquent behavior and substance use. Journal of Adolescent Health, 43, 15-22.

Hawkins, J. D., Oesterle, S., Brown, E. C., Arthur, M. W., Abbott, R. D., Fagan, A. A. y Catalano, R. F. (2009). Results of a type 2 translational research trial to prevent adolescent drug use and delinquency: A test of Communities That Care. Archives of Pediatrics and Adolescent Medicine, 163, 789-798. doi:10.1001/archpediatrics.2009.141.

Hawkins, J. D., Oesterle, S., Brown, E. C., Monahan, K. C., Abbott, R. D., Arthur, M. W. y Catalano, R. F. (2012). Sustained decreases in risk exposure and youth problem behaviors after installation of the Communities That Care prevention system in a randomized trial. Archives of Pediatrics Adolescent Medicine, 166, 141-148.

Hawkins, J. D., Oesterle, S., Brown, E. C., Abbott, R. D. y Catalano, R. F. (2014). Youth problem behaviors 8 years after implementing the Communities That Care prevention system: A community-randomized trial. JAMA Pediatrics, 168, 122-129.

Herrenkohl, T. I., Lee, J. O., Kosterman, R. y Hawkins, J. D. (2012) Family influences related to adult substance use and mental health problems: A developmental analysis of child and adolescent predictors. Journal of Adolescence Health, 51, 129-135. doi:10.1016/j.jadohealth.2011.11.003.

Jonkman, H. B., Haggerty, K. P., Steketee, M., Fagan, A. A., Hanson, K. y Hawkins, J. D. (2009). Communities That Care, core elements and context: Research of implementation in two countries. Social Developmental Issues, 30, 42-57.

Kenny, D. T. y Schreiner, I. (2009). Predictors of high-risk alcohol consumption in young offenders on community orders: Policy and treatment implications. Psychology, Public Policy, and Law, 15, 54-79. doi: 10.1037/a0015079.

Kilpatrick, D. G., Acierno, R., Saunders, B., Resnick, H. S., Best, C. L., y Schnurr, P. P. (2000). Risk factors for adolescent substance abuse and dependence: Data from a national sample. Journal of Consulting and Clinical Psychology, 68, 19-30.

Little, T. D., Rhemtulla, M., Gibson, K. y Schoemann, A. M. (2013). Why the items versus parcels controversy needn't be one. Psychological Methods, 18, 285-300.
Mallick, J. (2009). Parent drug education: A participatory action research study into effective communication about drugs between parents and unrelated young people. Drugs: Education, Prevention, and Policy, 14. 247-260. doi:10.1080/09687630601022416.

Medina, K., L. Schweinsburg, A. D., Cohen-Zion, M., Nagel, B. J. y Tapert, S. F. (2007). Effects of alcohol and combined marijuana and alcohol use during adolescence on hippocampal volume and asymmetry. Neurotoxicology, 29, 141-152.

Mejia-Trujillo, J., Pérez-Gómez A. y Reyes-Rodríguez, M. (2015). Implementación y adaptación en Colombia del sistema preventivo Communities That Care. Adicciones, 27, 253-264.

Ministerio de Justicia y del Derecho, Ministerio de Educación Nacional, y Ministerio de Salud y Protección Social. (2012). Estudio Nacional de Consumo de Sustancias Psicoactivas en Población Escolar. Recuperado de http://www. unodc.org/documents/colombia/Documentostecnicos/Estudio_Consumo_Escolares.pdf.

Moral-Jiménez, M. d. 1. V. y Ovejero, A. (2008). Experimentación con sustancias psicoactivas en adolescentes españoles: perfil de consumo en función de los niveles de edad. Revista Latinoamericana de Psicología, 41, 533-553.

Navarro Cicua, D. C., Méndez, M. y Muñoz, L. (2008). Factores en el consumo de alcohol en adolescentes. Pensamiento Psicológico, 4, 115-134.

Oesterle, S., Hawkins, J. D., Kuklinski, M. R., Fagan, A. A., Fleming, C., Rhew, I. C., ... Catalano, R. F. (2015). Effects of Communities That Care on males' and females' drug use and delinquency 9 years after baseline in a community-randomized trial. American Journal of Community Psychology, 56, 217-228.

Pérez-Gómez, A., Mejía-Trujillo, J., Brown, E. C. y Eisenberg, N. (2016). Adaptation and implementation of a science-based prevention system in Colombia: Challenges and achievements. Journal of Community Psychology, 44, 538-545. doi:10.1002/jcop.21781.

Pérez, A. y Scopetta, O. (2009). Consumo de alcohol en menores de 18 años en Colombia 2008: Estudio en 7 capitales y dos municipios pequeños. Bogotá: Nuevos Rumbos/Dupligráficas.

Sanz, M., Iraurgi, I., Martínez-Pampliega, A. y Cosgaya, L. (2006). Conflicto marital y consumo de drogas en los hijos. Adicciones, 18, 39-48.

Sawilowsky, S. S. (2009). New effect size rules of thumb. Journal of Modern Applied Statistical Methods, 8, 467-474.

Scoppetta, O., Pérez-Gómez, A. y Molano, C. M. (2011). Perfiles asociados al consumo de alcohol en adolescentes escolarizados mediante análisis de correspondencias múltpiles. Acta Colombiana de Psicología, 14, 139-146.

Sloboda, Z, Glantz, M. D. y Tarter, R. E. (2012) Revisiting the concepts of risk and protective factors for understanding the etiology and development of substance use and substance use disorders: Implications for preven- 
tion. Substance Use E् Misuse, 47, 944-962. doi:10.3109/1 0826084.2012 .663280 .

Sloboda, Z. (2005). Implications of epidemiologic information for effective drug abuse prevention strategies. In Z. Sloboda (Ed.), Epidemiology of Drug Abuse (pp. 211223). New York, NY: Springer.

StataCorp. (2013). Stata Statistical Software: Release 13. College Station, TX: StataCorp LP.

Thatcher, D. L. y Clark, D. B. (2008). Adolescents at risk for substance use disorders: Role of psychological dysregulation, endophenotypes, and environmental influences. Alcohol Research and Health, 31, 168-176.

Toumbourou, J. W. (1999). Implementing Communities That Care in Australia: A community Mobilisation approach to crime prevention. Trends E् Issues in Crime and Criminal Justice, 122, 1-6.

Wills, T. A., Bantum, E. O., Pokhrel, P., Maddock, J. E., Ainette, M. G., Morehouse, E. y Fenster, B. (2013). A dual-process model for early substance use: Tests in two diverse populations of adolescents. Health Psychology, 32, 533-542. doi:10.1037/a0027634.

Zeigler, D. W., Wang, C. C., Yoast, R. A., Dickinson, B. D., McCaffree, M. A., Robinowitz, C. B., ... Council on Scientific Affairs, American Medical Association. (2005). The neurocognitive effects of alcohol on adolescents and college students. Preventive Medicine, 40, 23-32. doi:10.1016/j.ypmed.2004.04.04. 